Revue européenne des sciences sociales

European Journal of Social Sciences

52-1| 2014

Politiques du libre accès en sciences humaines et sociales

\title{
Raymond BOUDON, 2012, Croire et savoir. Penser le politique, le moral et le religieux, Paris, PUF, 324 p.
}

\section{Emmanuel Picavet}

\section{(2) OpenEdition}

\section{Journals}

Electronic version

URL: http://journals.openedition.org/ress/2743

DOI: $10.4000 /$ ress. 2743

ISSN: $1663-4446$

\section{Publisher}

Librairie Droz

\section{Printed version}

Date of publication: 6 May 2014

Number of pages: $277-281$

ISBN: 978-2-600-01829-6

ISSN: 0048-8046

Electronic reference

Emmanuel Picavet, «Raymond BOUDON, 2012, Croire et savoir. Penser le politique, le moral et le religieux, Paris, PUF, 324 p. », Revue européenne des sciences sociales [Online], 52-1 | 2014, Online since 12 May 2014, connection on 22 September 2020. URL : http://journals.openedition.org/ress/2743 DOI : https://doi.org/10.4000/ress.2743

This text was automatically generated on 22 September 2020

(c) Librairie Droz 


\title{
Raymond BOUDON, 2012, Croire et savoir. Penser le politique, le moral et le religieux, Paris, PUF, $324 \mathrm{p}$.
}

\author{
Emmanuel Picavet
}

1 Dans cet ouvrage, Raymond Boudon réalise, en introduisant quelques nouveautés, une synthèse remarquable des thèmes qui apparaissaient déjà centraux dans plusieurs de ses ouvrages antérieurs, en particulier le grand ouvrage de 1995, Le Juste et le Vrai (Fayard), complété par une série d'études sur les sociologues classiques et une riche collection (en anglais notamment) d'études méthodologiques. Raymond Boudon réunit fermement un certain nombre de thèmes communs à la sociologie et à la philosophie, offrant différentes déclinaisons de la question de la justesse dans le jugement. L'auteur ne se limite pas, cependant, à la reprise ou à l'illustration des thèmes wébériens (dans une moindre mesure, tocquevilliens et durkheimiens) qui ont donné un tour original et affirmé à son œuvre sociologique. Les exemples empruntés à l'actualité ne sauraient davantage monopoliser l'attention du lecteur. L'essentiel, dans cette contribution nouvelle, est bien de rechercher une systématicité supérieure dans les analyses. On pourra apprécier, à cet égard, la finesse des remarques de l'auteur sur les rapports entre sociologie quantitative et études qualitatives centrées sur les raisons. La reconstruction des principes fondateurs du programme wébérien en sociologie (au-delà de Weber lui-même), au chap. I, est tout à fait magistrale. Elle peut se résumer par quelques postulats : les idées (et non les intérêts) sont la source de l'action humaine ; les causes des croyances sont des raisons consciemment aperçues; il y a une rationalisation des idées qui joue un rôle moteur dans la vie collective; les causes des croyances normatives sont des raisons pouvant entrer dans un processus de rationalisation (p. 39-41).

2 Raymond Boudon récuse les explications par trop mécaniques du social, dans lesquelles il voit des rameaux de la vieille théorie matérialiste de l'homme-machine (ici mise en valeur dans une perspective historique). On doit cependant remarquer que les explications qu'il propose, et plus encore les explications empruntées à des auteurs 
classiques et données en exemples pour la méthodologie, présentent aussi un caractère relativement mécanique. Elles empruntent souvent au registre de la compensation ou de l'équilibre. Par exemple, une différence de traitement de deux cas par ailleurs semblables attire l'attention: c'est qu'une différence doit exister sur un autre plan, expliquant l'écart constaté, comme on le voit dans la reprise au chap. VI d'un exemple d'Adam Smith : le bourreau, sans être qualifié ni gros travailleur, se trouve mieux payé que d'autres en compensation du caractère singulièrement répugnant de son activité. Les nombreux exemples d'explication convaincante empruntés aux pères fondateurs des sciences sociales se situent habituellement dans ce registre, exploitant autant que les sciences naturelles les ressources de la symétrie, de la compensation et de la restauration de l'équilibre.

3 Dans cette sociologie, corrélativement, les aspects symboliques et culturels jouent un rôle beaucoup plus discret que les aspects de la vie sociale qui se prêtent au calcul, aux échanges mesurés ou au discernement de symétries quantitatives ou comportementales. L'équilibre et la réciprocité occupent les premiers rôles, alors même que l'auteur fustige, dans une critique d'ensemble dit du « choix rationnel » en sociologie, les explications seulement fondées sur la recherche de l'intérêt ou les équilibres stratégiques. C'est sur cette ligne de faite que se situent les contributions récentes de Raymond Boudon à la pensée sociale, ici reprises, synthétisées et développées. D'un côté, les facteurs privilégiés dans les explications sont les mêmes que dans les théories du choix rationnel; d'un autre côté, ces dernières sont critiquées et dépassées en raison de leur concentration sur une forme de rationalité (l'optimisation au regard d'une synthèse des objectifs et des croyances) qui apparaitt trop proche de la seule « rationalité instrumentale».

C'est pourquoi les analyses donnent une place prépondérante à la forme rationnelle de l'action, de l'interaction et des explications qu'on en donne, plutôt qu'au contenu des motifs et des croyances. Toutefois, le rapport des agents à ce contenu (notamment aux coûts et aux avantages tels qu'on apprend à les établir, aux vérités telles qu'on peut les apercevoir à l'occasion de la formation des croyances) est reconnu comme un facteur essentiel de l'évolution sociale et l'approche de l'auteur - particulièrement intéressante à ce propos - s'inscrit en rupture à la fois par rapport au culturalisme (sous toutes ses formes) et par rapport au subjectivisme intégral trop fréquent dans les approches relevant du «choix rationnel». Certaines formules, comme celles qui concernent le déclin présumé de l'influence de l'État (ou du rôle de l'État dans le règne du droit) dans la mondialisation, au chap. IV, mériteraient sans doute d'être appréhendées en donnant toutes leurs chances à des approches fines du rôle symbolique et culturel de l'autorité publique dans l'aménagement des espaces de vie et dans le rapport aux normes et aux institutions.

5 La dénonciation insistante du culturalisme, qui s'inscrit dans une interrogation critique de l'emprise des idées relativistes, sert aussi le propos d'une recherche d'explications "sans reste ", tant pour les phénomènes sociaux directement observables que pour l'évolution des croyances et des convictions. Il s'agit en somme de contourner l'écueil d'explications tautologiques ou relevant de l'invocation de causes rendues inscrutables par une «boîte noire ». Néanmoins, on doit remarquer que l'entreprise critique visant le culturalisme pousse l'auteur à privilégier un type défini d'explications, certes moins étroitement circonscrit que dans les travaux fondés sur l'intérêt personnel: des 
explications privilégiant le traitement immédiat des personnes, la prise en compte impartiale de leurs intérêts et la comparaison de leurs situations respectives.

6 Le potentiel de la tradition sociologique (avec une mobilisation constante des thèmes durkheimiens) est appliqué à la question sans cesse retravaillée de l'explication des croyances religieuses, en redonnant du lustre à la thèse d'une association à portée explicative entre les croyances religieuses d'une part et les raisonnements ordinaires sur la morale et la condition humaine d'autre part (ch. II et ch. V). Dans ce champ, en remettant sur le métier les thèmes classiques de l'existence de l'âme immortelle et des récompenses ou sanctions de la justice divine, Raymond Boudon réaffirme le caractère explicatif des facteurs substantiels privilégiés par Max Weber ou Alexis de Tocqueville, tels que la congruence entre les impératifs des pratiques sociales ordinaires et le socle des croyances religieuses (contraste entre les pharisiens et les sadducéens au sujet de l'immortalité de l'âme, au chap. I) ou l'indépendance des contenus de la croyance par rapport aux conditions contextuelles de la vie sociale (à propos des caractéristiques du développement du christianisme en Afrique, au chap. V).

7 Certains aspects dynamiques sont pris en compte, en particulier ceux qui concernent la reconnaissance progressive des raisons les meilleures (lorsqu'elles existent et peuvent être identifiées, comme dans le cas du rejet progressif de la peine de mort ou de l'acceptation du suffrage universel), le reflux des erreurs démontrables, l'émergence des protestations bien justifiées et qui ne sont pas vouées à l'extinction faute de "privilège » au sens de la théorie olsonienne. Raymond Boudon rend d'ailleurs un hommage appuyé à cette dernière et cela pourra être remarqué, compte tenu du fait que l'analyse de la mobilisation collective chez Olson est souvent donnée comme l'un des principaux exemples de l'approche étroite - ici critiquée comme telle - du «choix rationnel ». D'autres aspects dynamiques de la vie sociale, en revanche, semblent voués à demeurer dans l'ombre - ou du moins dans un clair-obscur - dans les termes de la théorie proposée sous sa forme actuelle. On peut songer en particulier à ceux qui comportent des enjeux symboliques ou directement culturels tels que les identités collectives, malgré la détermination de l'auteur dans la recherche d'une "vérité du symbolique " (chap. V).

8 La théorie proposée semble également difficilement applicable telle quelle aux croyances qui, pour être particulièrement fragiles (voire franchement dépassées) n'en sont pas moins assorties d'un rôle fonctionnel auto-entretenu dans les échanges sociaux ou dans les transactions institutionnelles. Comment pourrait-elle rendre compte, par ailleurs, de la résistance résolue aux démarches d'universalisation ou de recherche d'impartialité ? Avec la théorie de Raymond Boudon, on parviendra sans difficulté à expliquer l'abandon de la ségrégation raciale; on aura plus de peine à rendre compte des progrès électoraux des partis populistes cultivant l'ambiguïté ou la double entente au regard du racisme et de l'antisémitisme. La théorie est directement applicable pour rendre compte de l'évolution des croyances au sujet de la démocratie représentative, en lien avec la morale universaliste de l'égale dignité humaine et de la recherche d'impartialité (chap. VI), mais elle serait certainement d'un maniement plus complexe s'il s'agissait de rendre compte de l'imbrication parfois conflictuelle des considérations impartiales et du souci de la promotion directe des intérêts (d'un individu, d'une sensibilité politique ou d'un parti).

9 En somme, la sociologie de Raymond Boudon n'est certainement pas loin d'être la meilleure dont nous disposions et elle influence fortement la «sociologie analytique » 
de Pierre Demeulenaere, à laquelle il est rendu hommage au chap. VII ; mais c'est une sociologie privilégiant les aspects du monde social dans lesquels la rationalisation wébérienne demeure en mouvement. C'est une sociologie dont l'objet même est largement rationnel, ce qui permet d'identifier sans abus « approche rationnelle » et théorie de l'acteur rationnel, d'une manière à coup sûr très pertinente pour de larges secteurs de la vie sociale. Elle appelle certainement des développements ultérieurs pour ce qui concerne la persistance ou le choix délibéré de l'obscurantisme, ou bien encore l'entretien soigneux des croyances dépassées dans les institutions. Il est bien possible que les tendances rationnelles éclairées par la théorie boudonienne imposent leur loi à très long terme ; mais que se passe-t-il dans les cadres sociaux persistants et avec les attitudes individuelles qui structurent le présent et l'avenir point trop éloigné ? Nous manquons certainement encore des repères théoriques qui nous permettraient d'apprécier de façon systématique, et si possible de mesurer, l'écart entre les raisons et arguments qui prévalent dans la vie sociale et ceux qui seraient corrects.

Par exemple, dans une société développée et éclairée telle que la société française, le rejet ordinaire de la garde alternée des enfants pour les parents séparés paraît traduire une violation persistante du principe d'égalité et de la reconnaissance égale de la contribution utile des parents à l'éducation des enfants, quel que soit leur sexe. Le travail de rationalisation ne semble pas s'effectuer, malgré l'existence de revendications claires et bien justifiées, reflétant les meilleures raisons disponibles et tendant à faire évoluer les pratiques. Pour autant, face à l'invocation ad hoc de raisons que les décisions prises contribuent à renforcer (comme dans le cas de décisions qui, sur leur volet financier, compromettent les chances d'accès du parent discriminé à un logement de taille adéquate), on ne peut guère avoir de doute quant à l'existence, à l'arrière-plan des décisions et en lien direct avec elles, de préférences ou de jugements de valeur, ou encore de croyances (et sans doute d'un mélange de ces facteurs). Les préjugés qui l'emportent en pratique traduisent des croyances ou représentations ancestrales. De fait, bien que les raisonnements individuels fassent partie des ressources explicatives des sciences sociales, il ne parait pas toujours possible de chercher à rationaliser les croyances ou les préférences. En cherchant à le faire malgré tout, on risque d'être déçu dans les cas où l'on devrait tout d'abord prêter attention au fait qu'il serait préférable, en termes rationnels, d'agir d'après des préférences ou des croyances autres que celles auxquelles les agents sociaux restent attachés en contexte, $\mathrm{du}$ fait de la sottise, de la paresse, de l'héritage culturel ou du conformisme. Rien en principe ne s'oppose ici au discernement de mécanismes qui, tout en s'éloignant d'une homologie entre les raisons de l'acteur et le meilleur jugement, peuvent être l'objet valable d'une science sociale analytique.

11 La sociologie de Raymond Boudon vise - non sans assise théorique et empirique - une portée explicative générale et ce motif est central dans la critique - instructive en ellemême - d'un large éventail d'approches concurrentes dans les sciences sociales. Le commentateur s'interroge alors inévitablement sur l'intérêt explicatif à attribuer aux aspects contextuels (locaux, temporaires, etc.) qui jouent un rôle si marqué dans nombre de phénomènes sociaux, et dont l'auteur lui-même a su mesurer l'importance. Ici, Raymond Boudon évoque le « paramétrage contextuel » qui permettrait d'appliquer les modèles explicatifs aux situations concrètes tout en préservant leur caractère authentiquement explicatif - sans tomber, donc, dans un ajustement ad hoc nous faisant revenir à la proverbiale "vertu dormitive» de l'opium. On peut cependant se demander si ce «paramétrage » ne voisine pas quelquefois avec un ajustement dans la 
théorie substantielle que l'on applique, dès lors que celle-ci vise bien, selon le vœu de l'auteur, à synthétiser les raisons qui sont à l'œuvre dans les croyances et la motivation des acteurs. Par exemple, au chap. VI, la diversité des causes du suicide est rapportée à la recherche de l'estime de soi, laquelle peut faire intervenir des facteurs très différents les uns des autres, comme un environnement oppressif ou une crise dans la vie personnelle. Cette variabilité des raisons à invoquer en fonction des circonstances, dont l'auteur fait un trait caractéristique de la manière dont les agents sociaux eux-mêmes procèdent dans l'élaboration de leurs raisons de croire et d'agir, peut aussi valoir justification d'une démarche ouverte à la diversité des tentatives théoriques et explicitement méthodologique dans ses ambitions.

Clarifier le format souhaitable des explications, voilà bien ce qui unifie les différentes analyses ici regroupées. L'ouvrage se laisse recommander tout à la fois par les analyses présentées et par un regard rétrospectif unique sur la tradition sociologique et sur les inflexions récentes dans les approches dites «compréhensives». Qu'il s'agisse du rapport entre théorie sociologique des croyances et philosophie du relativisme, ou encore de la classification des différentes approches naturalistes de l'explication sociologique, le lecteur ne pourra qu'être frappé par l'ampleur des perspectives et la richesse de l'argumentation. L'ouvrage offre donc tout à la fois une synthèse des apports personnels récents et un regard rétrospectif particulièrement précieux sur la sociologie d'inspiration wébérienne.

\section{AUTHORS}

\section{EMMANUEL PICAVET}

Université Paris-1 Panthéon-Sorbonne 